\title{
Serum Albumin Is Independently Associated with Persistent Organ Failure in Acute Pancreatitis
}

\author{
Wandong Hong, ${ }^{1}$ Suhan Lin, ${ }^{1}$ Maddalena Zippi, ${ }^{2}$ Wujun Geng, ${ }^{3}$ Simon Stock, \\ Zarrin Basharat, ${ }^{5}$ Bicheng Cheng, ${ }^{6}$ Jingye Pan, ${ }^{7}$ and Mengtao Zhou ${ }^{8}$ \\ ${ }^{1}$ Department of Gastroenterology and Hepatology, The First Affiliated Hospital of Wenzhou Medical University, \\ Wenzhou, Zhejiang, China \\ ${ }^{2}$ Unit of Gastroenterology and Digestive Endoscopy, Sandro Pertini Hospital, Rome, Italy \\ ${ }^{3}$ Department of Anesthesiology, The First Affiliated Hospital of Wenzhou Medical University, Wenzhou, Zhejiang, China \\ ${ }^{4}$ Department of Surgery, World Mate Emergency Hospital, Battambang, Cambodia \\ ${ }^{5}$ Microbiology \& Biotechnology Research Lab, Department of Environmental Sciences, Fatima Jinnah Women University, \\ Rawalpindi 46000, Pakistan \\ ${ }^{6}$ Zhejiang Provincial Top Key Discipline in Surgery, Wenzhou Key Laboratory of Surgery, Department of Surgery, \\ The First Affiliated Hospital, Wenzhou Medical University, Wenzhou, Zhejiang, China \\ ${ }^{7}$ Intensive Care Unit, The First Affiliated Hospital of Wenzhou Medical University, Wenzhou, Zhejiang, China \\ ${ }^{8}$ Department of Surgery, The First Affiliated Hospital of Wenzhou Medical University, Wenzhou, Zhejiang, China
}

Correspondence should be addressed to Mengtao Zhou; studyzhoumengtao@sina.com

Received 26 May 2017; Revised 4 September 2017; Accepted 11 September 2017; Published 24 September 2017

Academic Editor: Yousuke Nakai

Copyright (C) 2017 Wandong Hong et al. This is an open access article distributed under the Creative Commons Attribution License, which permits unrestricted use, distribution, and reproduction in any medium, provided the original work is properly cited.

\begin{abstract}
Background and Aims. To investigate the association between serum albumin levels within $24 \mathrm{hrs}$ of patient admission and the development of persistent organ failure in acute pancreatitis. Methods. A total of 700 patients with acute pancreatitis were enrolled. Multivariate logistic regression and subgroup analysis determined whether decreased albumin was independently associated with persistent organ failure and mortality. The diagnostic performance of serum albumin was evaluated by the area under Receiver Operating Characteristic (ROC) curves. Results. As levels of serum albumin decrease, the risk of persistent organ failure significantly increases $\left(P_{\text {trend }}<0.001\right)$. The incidence of organ failure was $3.5 \%, 10.6 \%$, and $41.6 \%$ in patients with normal albumin and mild and severe hypoalbuminaemia, respectively. Decreased albumin levels were also proportionally associated with prolonged hospital stay $\left(P_{\text {trend }}<0.001\right)$ and the risk of death $\left(P_{\text {trend }}<0.001\right)$. Multivariate analysis suggested that biliary etiology, chronic concomitant diseases, hematocrit, blood urea nitrogen, and the serum albumin level were independently associated with persistent organ failure. Blood urea nitrogen and the serum albumin level were also independently associated with mortality. The area under ROC curves of albumin for predicting organ failure and mortality were 0.78 and 0.87 , respectively. Conclusion. A low serum albumin is independently associated with an increased risk of developing of persistent organ failure and death in acute pancreatitis. It may also be useful for the prediction of the severity of acute pancreatitis.
\end{abstract}

\section{Introduction}

Though most patients with acute pancreatitis (AP) have a benign clinical course, approximately $10 \%-20 \%$ of patients develop persistent organ failure (defined as organ failure lasting for $\geq 48$ hours) associated with significant mortality of at least 30\% [1, 2]. Recent international consensus identified that persistent organ failure is the key determinant of severity regardless of the presence or absence of local pancreatic complications [3]. Early identification of patients likely to develop persistent organ failure would help physicians to select those patients who would benefit the most from close surveillance or aggressive intervention [4]. Existing scoring system such as the Bedside index of severity in acute pancreatitis (BISAP) [5] and the Harmless acute pancreatitis score (HAPS) [6] have moderate diagnostic accuracy in the prediction of persistent 
organ failure [2]. More recently, attention has also focused on assessing the association between persistent organ failure in acute pancreatitis and individual laboratory parameters such as admission hematocrit $\geq 44 \%$, rise in blood urea nitrogen (BUN) at 24 hours [7], serum triglycerides [8], and highdensity lipoprotein [9].

Albumin is exclusively synthesized in the liver. Low serum albumin $(<35 \mathrm{~g} / \mathrm{L})$ is often observed in hospitalized elderly patients [10] or patients with decompensated liver cirrhosis, malnutrition, nephrotic syndrome, diabetes, heart failure, cancer, and sepsis [11, 12]. Hypoalbuminemia has been proposed as a useful predictor of morbidity and mortality in many different clinical settings such as community-acquired pneumonia [12] and hemodialysis and coronary heart disease [13]. As a negative acute phase protein, initial serum albumin was also independently associated with disease progression to severe sepsis [14] and 30-day mortality in emergency medical patients, irrespective of the cause $[15,16]$.

A few studies have evaluated hypoalbuminemia as predictor of severe acute pancreatitis [17-19]. However, these studies are limited due to small sample size with low statistical power and inconsistent definitions of severity ranging from severe pancreatitis based on the Atlanta criteria to mortality. Although a recent study by Li et al. [20] had used persistent organ failure of acute pancreatitis as the primary outcome, the results of that study [20], as well as the above-mentioned studies [17-19], are biased due to lack of adjustment for confounding factors such as etiology and chronic concomitant diseases. Furthermore, to the best of our knowledge, no study has performed a complete overview of the association between serum albumin levels at admission and outcomes in acute pancreatitis up until now. Therefore, the aim of this study was to investigate whether serum albumin levels within 24 hrs of patient admission correlate with clinical outcomes in acute pancreatitis.

\section{Materials and Methods}

Patients with AP who were admitted (index admissions) to our hospital within 72 hours of the onset of symptoms between January 2012 and January 2015 were enrolled for the study. Acute pancreatitis was defined as described previously [4]. Organ failure [1] was defined as having a Marshall score $\geq$ 2 for at least one of the three organs (respiratory, cardiovascular, and renal failure) involved. Duration of organ failure was defined as persistent if it lasted for $>48$ hours $[1,3]$. Exclusion criteria included [21] patients that had developed organ failure before data collection, recurrent or not first-time pancreatitis, previous pancreatic surgery, ERCP or traumainduced pancreatitis, chronic pancreatitis, pancreatic cancer, pleural effusions preceding the development of AP, and pleural effusions resulting from concomitant diseases (e.g., pneumonia, chronic heart failure), patients with albumin infusion before data collection in our hospital, hypoalbuminemia due to malnutrition, chronic renal disease, albuminuria, hepatitis, bleeding/coagulation disorders, chronic alcoholism, and liver cirrhosis, and patients for whom completed data was unavailable. Chronic concomitant diseases [12] were classified as neurologic (stroke), cardiovascular (coronary heart disease and arrhythmia), pulmonary (emphysema and chronic bronchitis), diabetes mellitus, hypertension, hepatitis virus carrier, and fatty liver. This study protocol was approved by the Ethics Committee of the First Affiliated Hospital of Wenzhou Medical College.

Age, gender, body mass index (BMI), time from pain onset to admission, and biochemical parameters were recorded within 12 hours of admission before the development of persistent organ failure [4, 21]. Serum albumin levels were measured within 24 hours of admission [12]. If patients had multiple albumin measurements within 24 hrs, only the first-time measurement was picked. Hypoalbuminemia was defined by a serum albumin $<35 \mathrm{~g} / 1$ [11]. Similar to previous studies $[8,22]$, patients with hypoalbuminemia were further divided into mild $(<35 \mathrm{~g} / \mathrm{l}$ but $\geq 30 \mathrm{~g} / \mathrm{l})$ and severe $(<30 \mathrm{~g} / \mathrm{l})$ groups according to the serum albumin level.

2.1. Statistical Analysis. Sample size calculation was based on identifying independent dichotomous predictors in a multivariable logistic regression analysis for persistent organ failure [23]. With an $\alpha$ risk of 0.05 ( $\alpha$ is the probability of rejecting a true null hypothesis) and $\beta$ risk of $0.2(\beta$ is the probability of accepting a false null hypothesis), and a bilateral test, the sample size was calculated for the following hypotheses: assuming a prevalence of persistent organ failure at $10 \%$ and a low correlation between the predictor and other covariates $\left(R^{2}=0.10\right)(R$-squared, namely, the coefficient of determination, which is equal to the squared correlation coefficient and provides an estimate of the percent of variation in one variable that is explained by the other variables [24]). A sample of 668 patients would provide $80 \%$ power of detecting an adjusted odds ratio of 2.0 for a predictor with an overall prevalence of $35 \%$.

A Shapiro-Wilk test was used to evaluate whether the continuous data was a normal distribution. According to the results of Shapiro-Wilk test, continuous values were expressed by mean \pm standard deviation (SD) or median and interquartile range (IQR) and compared using one-way analysis of variance or the Kruskal-Wallis nonparametric test. Categorical values were described by count and proportions and compared by the $\chi^{2}$ test or Fisher's exact test.

Linear trend of categorical and continuous variables was tested by a Royston extension of the Cochran-Armitage test [25] and a nonparametric Wilcoxon rank sum test [26], respectively.

Univariate logistic regression analysis was used to identify potential independent predictors of persistent organ failure and death. Except for serum albumin level and the time from pain onset to admission [8], all other variables, namely, age ( $\geq 60$ years) [8], male sex [27], BMI $(\geq 30)$ [28], biliary etiology (yes versus no), chronic concomitant diseases (yes versus no) [12], hematocrit ( $\geq 44 \%)$ [7], total bilirubin ( $\geq 2 \mathrm{mg} / \mathrm{dL})$, alanine aminotransferase (ALT > 50 units/L) [29], glucose $(\geq 150 \mathrm{mg} / \mathrm{dL})$ [28], and blood urea nitrogen (BUN) (>25 mg/dL) [5], were used as dichotomous/categorical variable when performing logistic regression analysis. Variables reaching statistical difference among patients with different albumin levels or between patients with and without organ failure in univariate logistic regression analysis were included 


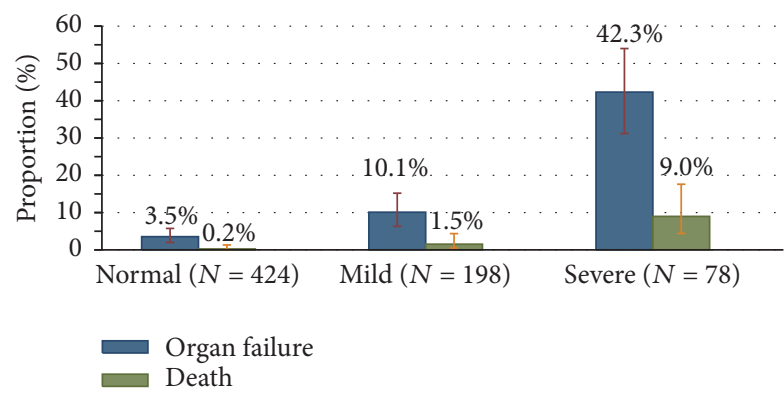

(a)

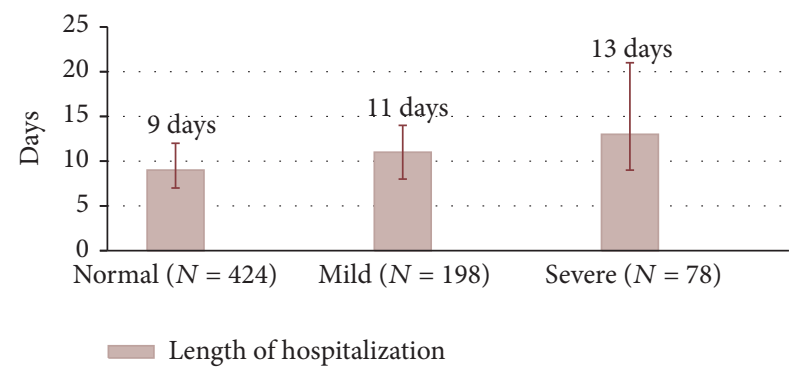

(b)

FIGURE 1: Trends of median hospital days, incidence of persistent organ failure, and mortality in acute pancreatitis patients with normal, mild, and severe low serum albumin.

in the multivariate logistic regression analysis. Multivariate logistic regression was used to evaluate the relationship between patients with different albumin levels and persistent organ failure and death adjusted for potential risk factors and confounding factors. Odds ratios (OR) were calculated, with 95\% CI [30]. Multicollinearity was considered to be significant if the largest variance inflation factor exceeded 10 [4].

The area under the Receiver Operating Characteristic Curve (AUC) was used to evaluate the performance of predictions. The serum albumin level was used as a continuous rather than categorical variable when performing Receiver Operating Characteristic (ROC) curve analysis. A variable with an AUC above 0.7 was considered useful, while an AUC between 0.8 and 0.9 indicated excellent diagnostic accuracy [31]. As described by Maksimow et al. [32], the best cut-off point was selected based on that where the number of false positives is as low as possible (specificity $>90 \%$ ) by selecting a threshold value at a point where the longest increase in the sensitivity of the slope declines since ICU beds are limited. The sensitivity, specificity, negative predictive value, positive predictive value, positive likelihood ratio, negative likelihood ratio, and diagnostic accuracy were calculated for corresponding cut-off values.

Differences were considered to be statistically significant if the two-tailed $P$ value was less than 0.05 .

\section{Results}

3.1. Characteristics of Study Subjects. A total of 700 patients were included in the study. 435 (62.1\%) were male, with a median age of 48 (IQR 37-63) years. The mean time interval between onset and admission was $1.8 \pm 0.8$ days. The median value of albumin within $24 \mathrm{hrs}$ of admission was $36.2 \mathrm{~g} / \mathrm{L}$ (IQR: 32.9-39.7). The overall prevalence of hypoalbuminemia $(<35 \mathrm{~g} / \mathrm{L})$ was 39.4\% (276/700). Of these, 198 had mild hypoalbuminemia, while 78 had severe hypoalbuminemia.

In all the patients, the most common etiology of AP was biliary (42.7\%). 24 patients had combined biliary and alcohol etiology and 9 patients had combined biliary and hypertriglyceridemia etiology. Nearly 57.4\% (402/700) of patients had $\geq 1$ comorbidities, mainly fatty liver (257/700, $36.7 \%)$, hypertension $(160 / 700,22.9 \%)$, and diabetes mellitus (105/700, 15.0\%). 68 (9.7\%) patients developed persistent OF, which was multiple $(\geq 1) \mathrm{OF}$, in 31 (45.6\%) patients. Respiratory failure (82.4\%) was the most frequent OF. Eleven (1.6\%) patients with OF died from AP, seven during the first week in hospital.

3.2. Clinical Features and Results of Patients with Different Serum Albumin Levels. The demographic, clinical, and laboratory findings at hospital admission of patients with different serum albumin levels are shown in Table 1. A greater increase in age $\left(P_{\text {trend }}=0.012\right)$ and longer time from pain onset to admission $\left(P_{\text {trend }}<0.001\right)$ were associated with decreased serum albumin level. No significant difference was observed among patients with different albumin levels with respect to gender, BMI, and alcohol etiology.

There was also a trend for increasing rates of overall incidence of chronic concomitant diseases with a lower serum albumin level though it did not reach statistical significance $(P=0.134)$. However, there was a significant statistical difference among patients with different albumin levels with respect to biliary etiology and concomitant neurologic disease though it occurred in a few patients. Regarding laboratory findings, decline in serum albumin level was associated with a decrease in hematocrit $(P=0.001)$, alanine aminotransferase $(P=0.001)$, glucose $(P=0.003)$, and BUN $(P<0.001)$.

As shown in Table 1 and Figure 1, persistent organ failure developed in 3.5\% (15/424) patients with normal albumin, $10.1 \%(20 / 198)$ patients with mild hypoalbuminemia, and $42.3 \%$ (33/78) with severe hypoalbuminemia when patients were divided into three groups according to different albumin levels. This indicated that a greater decrease in serum albumin level was associated with increased organ failure rate $\left(P_{\text {trend }}<\right.$ 0.001). There was also a statistically significant trend for increasing rates of mortality $\left(P_{\text {trend }}<0.001\right)$ and prolonged hospital stay $\left(P_{\text {trend }}<0.001\right)$ with a lower serum albumin level. 
TABLE 1: Baseline clinical characteristics and outcomes among patients with different albumin levels.

\begin{tabular}{|c|c|c|c|c|}
\hline \multirow[b]{2}{*}{ Characteristic } & \multirow[b]{2}{*}{$\begin{array}{c}\text { Normal } \\
(N=424)\end{array}$} & \multicolumn{2}{|c|}{ Hypoalbuminemia } & \multirow[b]{2}{*}{$P$ value } \\
\hline & & $\begin{array}{c}\text { Mild } \\
(N=198)\end{array}$ & $\begin{array}{c}\text { Severe } \\
(N=78)\end{array}$ & \\
\hline Median age, years (IQR) & $46.5(37-60)$ & $50(39-67)$ & $47.5(36-70)$ & 0.012 \\
\hline Male sex, $N(\%)$ & $273(64.4)$ & $116(58.6)$ & $46(59.0)$ & 0.316 \\
\hline Time from pain onset to admission (days) & $1.75 \pm 0.77$ & $1.88 \pm 0.79$ & $2.10 \pm 0.82$ & $<0.001$ \\
\hline $\mathrm{BMI} \geq 30, N(\%)$ & $23(5.4)$ & $12(6.1)$ & $4(5.1)$ & 0.934 \\
\hline \multicolumn{5}{|l|}{ Etiology ${ }^{\star}$} \\
\hline Biliary, $N(\%)$ & $198(46.7)$ & $87(43.7)$ & $23(30.0)$ & 0.024 \\
\hline Alcohol, $N(\%)$ & $56(13.2)$ & $29(14.6)$ & $11(14.3)$ & 0.888 \\
\hline Hypertriglyceridemia, $N(\%)$ & $21(5.0)$ & $8(4.0)$ & $8(10.1)$ & 0.094 \\
\hline Idiopathic, $N(\%)$ & $145(34.2)$ & $68(34.2)$ & $33(42.9)$ & 0.323 \\
\hline Other, $N(\%)$ & $9(2.1)$ & $9(4.5)$ & $4(5.2)$ & 0.153 \\
\hline Chronic concomitant diseases & $240(56.6)$ & $116(58.3)$ & $53(68.8)$ & 0.134 \\
\hline Neurologic, $N(\%)$ & $4(0.9)$ & $1(0.5)$ & $5(0.5)$ & 0.004 \\
\hline Cardiovascular, $N(\%)$ & $13(3.1)$ & $13(6.5)$ & $4(5.2)$ & 0.126 \\
\hline Pulmonary, $N(\%)$ & $5(1.2)$ & $4(2.0)$ & $2(2.6)$ & 0.385 \\
\hline Diabetes mellitus, $N(\%)$ & $66(15.6)$ & $27(13.6)$ & $12(15.6)$ & 0.800 \\
\hline Hypertension, $N(\%)$ & $92(21.7)$ & $48(24.1)$ & $20(26.0)$ & 0.365 \\
\hline Hepatitis virus carrier, $N(\%)$ & $16(3.8)$ & $7(3.5)$ & $2(2.6)$ & 0.721 \\
\hline Fatty liver, $N(\%)$ & $162(38.2)$ & $63(31.7)$ & $27(35.1)$ & 0.279 \\
\hline \multicolumn{5}{|l|}{ Laboratory findings } \\
\hline Hematocrit & $0.43(0.38-0.46)$ & $\begin{array}{c}0.41 \\
(0.37-0.45)\end{array}$ & $\begin{array}{c}0.40 \\
(0.37-0.45)\end{array}$ & 0.001 \\
\hline Bilirubin, mg/dL (IQR) & $1.17(0.82-1.70)$ & $\begin{array}{c}1.23 \\
(0.82-1.81)\end{array}$ & $\begin{array}{c}1.053 \\
(0.64-1.81)\end{array}$ & 0.805 \\
\hline ALT, U/L (IQR) & $43(21-160)$ & $34(16-92)$ & $36(19-57)$ & 0.001 \\
\hline Glucose, mg/dL (IQR) & $139(114-182)$ & $150(119-200)$ & $171(117-216)$ & 0.003 \\
\hline BUN, mg/dL (IQR) & $12.7(10.1-16.2)$ & $\begin{array}{c}15.1 \\
(11.2-19.6)\end{array}$ & $\begin{array}{c}16.0 \\
(10.6-29.4)\end{array}$ & $<0.001$ \\
\hline \multicolumn{5}{|l|}{ Outcomes } \\
\hline Median hospital days (IQR) & $9(7-12)$ & $11(8-14)$ & $13(9-21)$ & $<0.001$ \\
\hline Organ failure $N(\%)$ & $15(3.5)$ & $20(10.1)$ & $33(42.3)$ & $<0.001$ \\
\hline Death, $N(\%)$ & $1(0.2)$ & $3(1.5)$ & $7(9.0)$ & $<0.001$ \\
\hline
\end{tabular}

${ }^{\star}$ Subjects may have more than one etiology. Subjects may have more than one chronic concomitant disease.

3.3. Independent Predictors of Organ Failure and Mortality. As shown in Figure 2, univariate analysis revealed that biliary etiology, chronic concomitant diseases, hematocrit, glucose, BUN, and the serum albumin level were significantly associated with the development of persistent organ failure. Multivariable logistic regression identified biliary etiology (OR: $0.44 ; 95 \%$ CI: $0.22-0.87 ; P=0.018$ ), chronic concomitant diseases (OR: 5.26; 95\% CI: 2.07-11.31; $P<0.001$ ), hematocrit $\geq 44 \%$ (OR: 2.92; 95\% CI: $1.55-5.51 ; P=0.001$ ), BUN > 25 mg/dL (OR: 8.30; 95\% CI: 4.05-17.01; $P<0.001$ ), and the serum albumin level (OR: $0.81 ; 95 \%$ CI: $0.76-0.87$; $P<0.001)$ were independently associated with persistent organ failure.

As shown in Figure 3, factors considered potentially relevant to mortality were analyzed by univariate and multivariate logistic regression. Multivariate logistic regression identified that BUN > 25 mg/dL (OR: 5.06; 95\% CI: 1.37-18.67; $P=0.015$ ) and the serum albumin level (OR: 0.79; $95 \%$ CI: $0.69-0.91 ; P=0.001)$ were independently associated with mortality. Multicollinearity between covariates did not exist, and the variance inflation factor was less than 10 for all variables.

3.4. Subgroup Analysis. Subgroup analysis confirmed the strong association between the serum albumin level and persistent organ failure across subgroups of age, gender, the time from pain onset to admission, different etiology, chronic concomitant diseases, total bilirubin, ALT, hematocrit, glucose, and BUN (Figure 4). As to association between the serum albumin level and mortality, subgroup analysis confirmed that these associations were stronger in subgroups of patients with one day from pain onset to admission, 


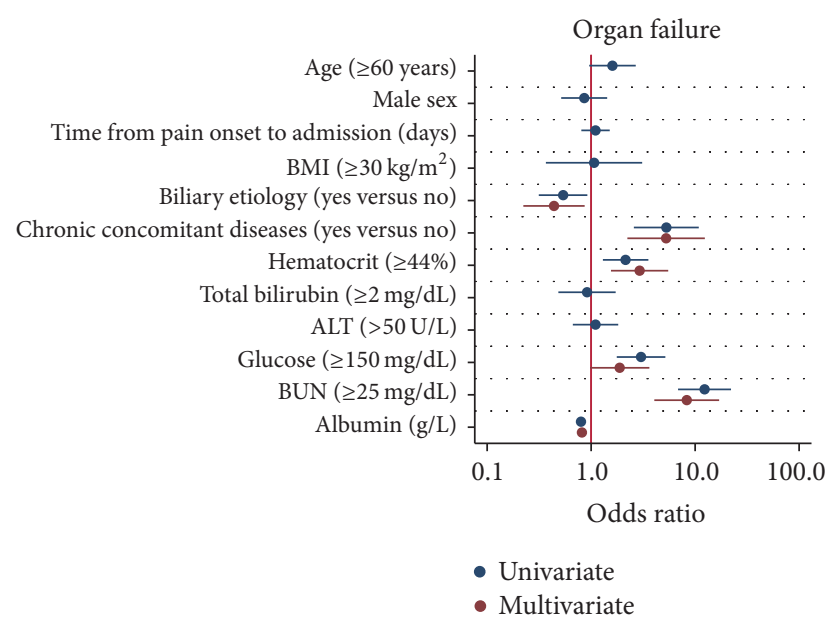

FIGURE 2: Univariate and multivariate logistic regression plot of odds ratios and 95\% confidence intervals for evaluation of the relationship between serum albumin level and persistent organ failure in acute pancreatitis.

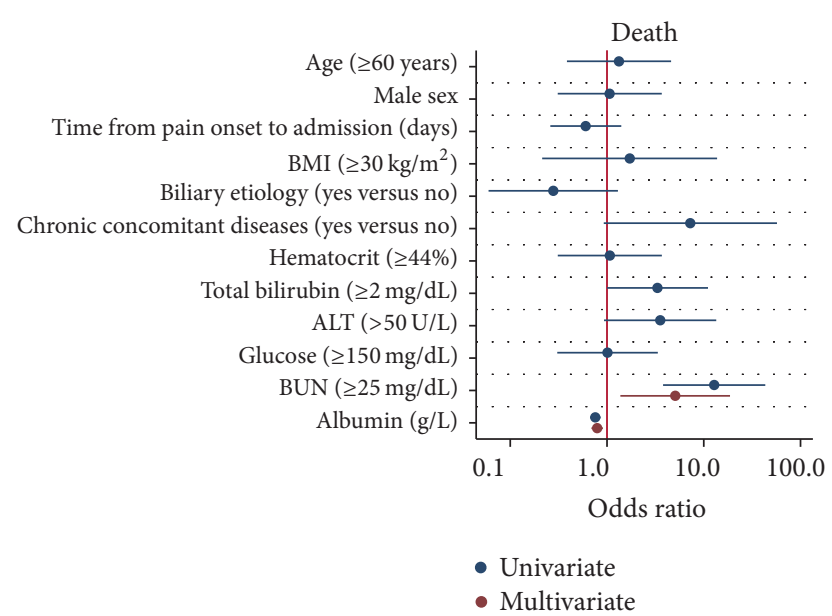

FIGURE 3: Univariate and multivariate logistic regression plot of odds ratios and 95\% confidence intervals for evaluation of the relationship between serum albumin level and death in acute pancreatitis.

nonbiliary etiology, and chronic concomitant diseases than those in subgroups with two or three days from pain onset to admission and biliary etiology and without chronic concomitant diseases (Figure 5).

3.5. Albumin as Early Predictor of Persistent Organ Failure and Mortality. The ROC curve for albumin for the prediction of persistent organ failure and mortality is shown in Figure 6. The AUC of albumin for predicting organ failure and mortality were 0.78 (95\% CI: $0.72-0.85)$ and 0.87 (95\% CI: 0.78-0.95), respectively. As shown in Table 2, the best cutoff points to predict organ failure and mortality were albumin level $\leq 30.8 \mathrm{~g} / \mathrm{L}$ and $\leq 29 \mathrm{~g} / \mathrm{L}$, respectively.

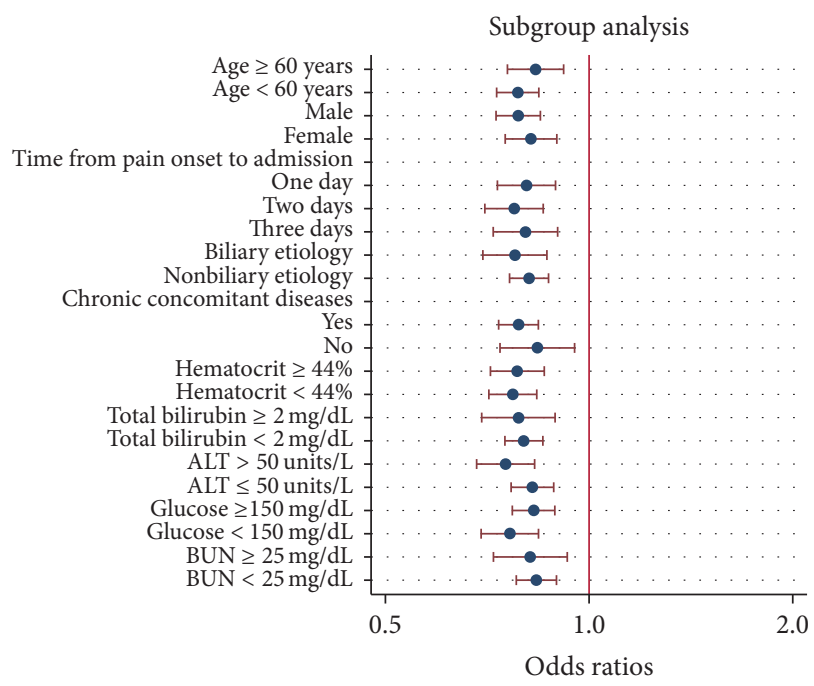

FIGURE 4: Logistic regression plot of odds ratios and 95\% confidence intervals; subgroup analysis of relationship between serum albumin level and risk of persistent organ failure in acute pancreatitis.

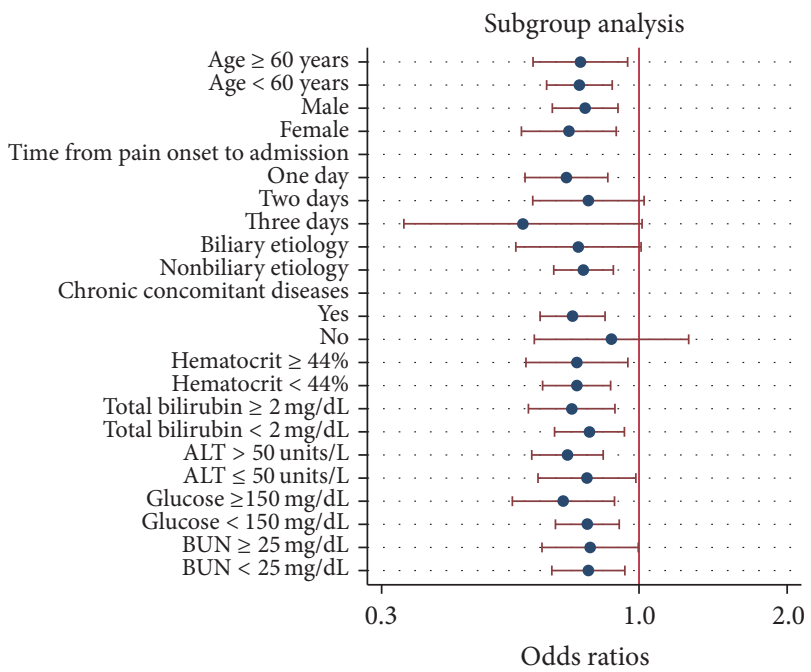

FIGURE 5: Logistic regression plot of odds ratios and 95\% confidence intervals; subgroup analysis of relationship between serum albumin level and mortality in acute pancreatitis.

\section{Discussion}

The results of the present study demonstrated the following: (i) persistent organ failure and early hypoalbuminemia occurred in $9.7 \%$ and $39.3 \%$ of patients with acute pancreatitis, respectively; (ii) as a risk factor, low serum albumin levels within 24 hours of admission are independently associated with the development of persistent organ failure and mortality in acute pancreatitis. Furthermore, this association is proportional; meaning the lower the serum albumin level, the greater the likelihood for persistent organ failure and mortality. The associations between the serum albumin level and mortality were stronger in subgroups of patients with one day from pain onset to admission, nonbiliary etiology, 
TABLE 2: The best cut-off values of albumin levels to predict organ failure and death in patients with acute pancreatitis.

\begin{tabular}{lcc}
\hline & Organ failure & Death \\
\hline AUC (95\% CI) & $0.78(95 \%$ CI: $0.72-0.85)$ & $0.87(95 \%$ CI: $0.78-0.95)$ \\
Albumin level (cutoff point) & $\leq 30.8 \mathrm{~g} / \mathrm{L}$ & $\leq 29 \mathrm{~g} / \mathrm{L}$ \\
Sensitivity & 0.53 & 0.55 \\
Specificity & 0.90 & 0.92 \\
Positive likelihood ratio & 5.40 & 6.83 \\
Negative likelihood ratio & 0.52 & 0.49 \\
Positive predictive value & 0.37 & 0.10 \\
Negative predictive value & 0.95 & 0.99 \\
Diagnostic accuracy & 0.87 & 0.91 \\
\hline
\end{tabular}

$\mathrm{AUC}=$ area under the curve of the Receiver Operating Characteristic Curve.

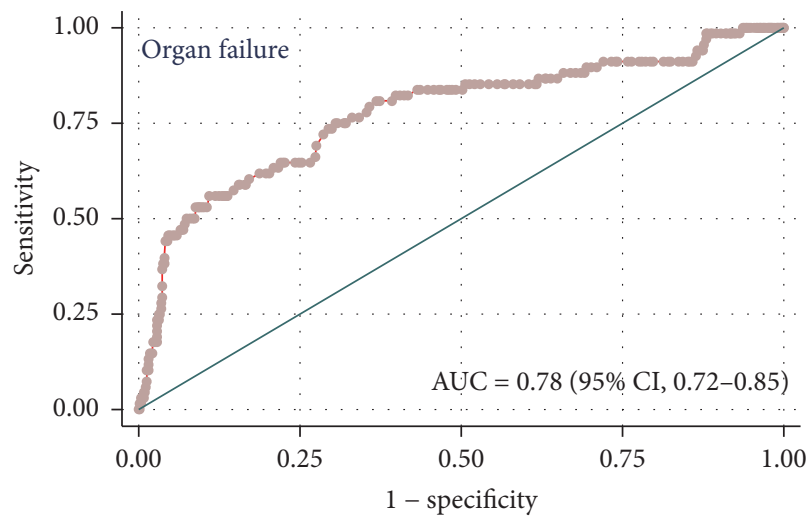

(a)

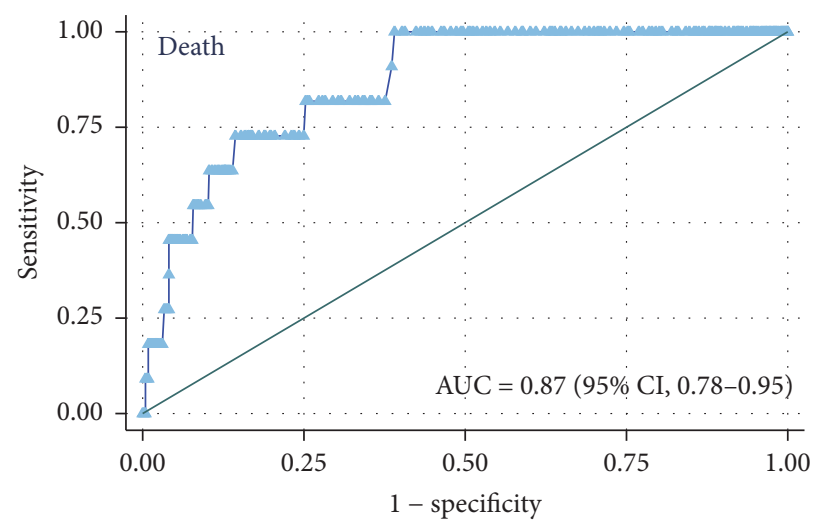

(b)

FIGURE 6: Receiver operating characteristic curves for serum albumin to predict the development of persistent organ failure and occurring of death in acute pancreatitis.

and chronic concomitant diseases than those in subgroups with two or three days from pain onset to admission and biliary etiology and without chronic concomitant diseases; (iii) as a predictor, the serum albumin had moderate and excellent performance for prediction of persistent organ failure (AUC: 0.78) and mortality (AUC: 0.87) in acute pancreatitis, respectively.

The mechanisms of hypoalbuminemia in acute pancreatitis are diverse [11, 33, 34]: (i) a decrease in energy or amino acid supply due to fasting and increased tissue catabolism in acute pancreatitis, (ii) excessive release of inflammatory cytokines such as interleukin-1, interleukin6 , and tumor necrosis factor $\alpha$ in the early stage of acute pancreatitis resulting in a decreased albumin synthesis in the liver, and (iii) active inflammatory cytokines also injuring microvascular endothelial cells which result in increased capillary permeability leading to a redistribution of albumin from the intravascular to the interstitial space.

Albumin has many physiologic roles including maintaining osmotic pressure, protecting the microvasculature and mitigating increased vascular permeability, binding of endogenous and exogenous substances, antioxidant and scavenging free radicals, anticoagulant effect, maintenance of acid base status, anti-inflammatory effects, and antiapoptosis
$[12,33]$. Therefore, hypoalbuminemia may be causally linked to the development of persistent organ failure rather than serving simply as a marker of severity of acute pancreatitis. The persistent organ failure in acute pancreatitis mainly includes respiratory, cardiovascular, and renal failure [3]. Albumin may improve oxygenation in patients with acute respiratory distress syndrome by reduced alveolarcapillary permeability, decreased inflammatory cell infiltration, enhanced total plasma antioxidant capacity, and faster hemodynamic stabilization $[35,36]$. A study in chronic obstructive pulmonary disease suggested that hypoalbuminemia is independently associated with the development of acute respiratory failure [37]. On the other hand, hypoalbuminemia is associated with decreased colloid osmotic pressure, which can lead to the development of pulmonary edema and exacerbation of acute heart failure [38]. The causal relationship between hypoalbuminemia and acute kidney injury is now confirmed [39]. Albumin may improve renal perfusion and glomerular filtration through prolonged renal vasodilation $[40,41]$, stimulation of the proliferation of proximal renal tubular epithelial cells by activation of mitogenic pathways [42], and inhibition of apoptosis of renal tubular cells by scavenging of reactive oxygen species [43]. Therefore, the fact that hypoalbuminemia is associated with 
prolonged hospital stay and increased morbidity and mortality has been noted in many clinical scenarios regardless of the implicated disease $[10,11,13,15,16,27]$. On the other hand, positive protective effects of human albumin solutions have been noted in several clinical scenarios such as septic shock [44] and liver cirrhosis with hepatorenal syndrome [45] or with spontaneous bacterial peritonitis, a disease that shares important pathophysiological features with severe sepsis [46]. It is unknown whether administration of albumin would improve the clinical outcomes of acute pancreatitis with hypoalbuminemia though severe acute pancreatitis has many similarities to sepsis syndrome and septic shock [47]. Therefore, it would be interesting to conduct a randomized clinical trial to assess the effect of the correction of hypoalbuminemia on acute pancreatitis in the future.

A few reports have also attempted to study the effect of serum albumin levels on the clinical outcomes of acute pancreatitis. Xue et al. [18] suggested that hypoalbuminemia in the early stage can accelerate the deterioration in pathophysiology of severe acute pancreatitis and it was associated with a high incidence of infection and mortality. GonzalvezGasch et al. [19] use albumin $<25 \mathrm{~g} / \mathrm{L}$ as a point in the prediction score for complicated acute pancreatitis. A recent study by Li et al. [20], which used persistent organ failure as primary outcome, proposed that serum albumin was a good indicator of persistent organ failure in acute pancreatitis. However, all the above-mentioned have small sample sizes with low statistical power and the results were not adjusted for confounding factors such as age, gender, the time from pain onset to admission, etiology, and chronic concomitant disorders. In addition, no subgroup analysis was performed in these studies. Therefore, these studies were not comparable to our current study. The current study showed that the risk of persistent organ failure significantly increases as levels of serum albumin decrease $\left(P_{\text {trend }}<0.001\right)$ (Figure 1). Decreased albumin levels were also proportionally associated with prolonged hospital stay $\left(P_{\text {trend }}<0.001\right)$ and an increased risk of mortality $\left(P_{\text {trend }}<0.001\right)$. Multivariate logistic regression analysis suggested that an increase of $1 \mathrm{~g} / \mathrm{L}$ in serum albumin level was associated with a statistically significant $19 \%$ (OR: 0.81 ; 95\% CI: $0.76-0.87$ ) reduction in the odds of persistent organ failure after adjusting for potential confounders (Figure 2). Similarly, multivariate logistic regression analysis suggested that an increase of $1 \mathrm{~g} / \mathrm{L}$ in serum albumin level was associated with a statistically significant 21\% (OR: $0.79 ; 95 \%$ CI: $0.69-0.91 ; P=0.001$ ) reduction in the odds of mortality after adjusting for potential confounders (Figure 3). Subgroup analysis confirmed the strong association between the serum albumin level and persistent organ failure across subgroups of age, gender, the time from pain onset to admission, different etiology, chronic concomitant diseases, total bilirubin, ALT, hematocrit, glucose, and BUN (Figure 4). As to association between the serum albumin level and mortality, subgroup analysis confirmed that these associations were stronger in subgroups of patients with one day from pain onset to admission, nonbiliary etiology, and chronic concomitant diseases than in those subgroups with two or three days from pain onset to admission and biliary etiology and without chronic concomitant diseases (Figure 5).

The area under ROC curves of albumin for predicting organ failure and mortality were 0.78 (95\% CI: 0.72-0.85) and 0.87 (95\% CI: 0.78-0.95), respectively (Figure 6). These results suggest that albumin may be used as an early predictor of persistent organ failure and mortality. As shown in Table 2, with a cut-off value of $\leq 30.8 \mathrm{~g} / \mathrm{L}$, albumin had a sensitivity of $53 \%$ and a specificity of $90 \%$ with an overall accuracy of $87 \%$ for prediction of organ failure. With a cut-off value of $\leq 29 \mathrm{~g} / \mathrm{L}$, albumin had a sensitivity of 55\% and a specificity of $91 \%$ with an overall accuracy of $93 \%$ for prediction of death. Both cutoff values had low sensitivity, which means that some of these patients who were at high risk of developing persistent organ failure and death may not have a low serum albumin level. However, both cut-off values had high negative predictive value, which is important in emergency for identification of patients not at risk for the development of persistent organ failure and death.

The existing scoring systems have shown moderate accuracy in predicting persistent organ failure but are cumbersome to use in the clinical setting [2]. Xu et al. [48] suggested that fatty liver assessed by computed tomography was associated with severity of acute pancreatitis. Mikolasevic et al. [49] showed that presence of nonalcoholic fatty liver was related to more severe forms of acute pancreatitis. Therefore, it is no surprise that chronic concomitant disease (OR: 5.26; 95\% CI: 2.07-11.31) was independently associated with persistent organ failure since fatty liver was the most common chronic concomitant disease in our study (Table 1). Cho et al. [50] reported that patients with alcoholic acute pancreatitis have a longer hospital stay and higher incidence of persistent organ failure compared to those with biliary acute pancreatitis. As expected, our results indicated that patients with biliary etiology had a statistically significant 56\% (OR: 0.44 ; 95\% CI: $0.22-0.87$ ) reduction in the odds of persistent organ failure compared to patients without biliary etiology. Simple, routine biomarkers such as hematocrit [7] and blood urea nitrogen (BUN) [7, 51-53] have been proposed as markers of disease severity. Koutroumpakis et al. [7] suggested that admission hematocrit $\geq 44 \%$ and rise in BUN at $24 \mathrm{hrs}$ may be the optimal predictive tools in clinical practice among existing laboratory parameters and scoring systems. Mounzer et al. [2] showed that BUN had similar accuracy in comparison with more complex scoring systems in predicting organ failure. As expected, our results suggested that hematocrit $\geq 44 \%$ (OR: $2.92 ; 95 \%$ CI: $1.55-5.51$ ) and BUN > $25 \mathrm{mg} / \mathrm{dL}$ (OR: 8.30; 95\% CI: 4.05-17.01) were independently associated with persistent organ failure. BUN $>25 \mathrm{mg} / \mathrm{dL}$ was also associated with a higher mortality risk in acute pancreatitis compared to patients with BUN $\leq 25 \mathrm{mg} / \mathrm{dL}$ (OR: 5.06; 95\% CI: 1.37-18.67; $P=0.015$ ), which is consistent with a previous report [5]. Additionally, contrary to the report by Kumaravel et al. [54], our study showed that BMI was not associated with organ failure and mortality in acute pancreatitis. These differences may be due to the variations among studies regarding the proportion of different etiology and number of obese patients, as evidenced by the fact that only a small proportion of patients with alcohol etiology and 
obesity were enrolled in our study (Table 1). The drawbacks in currently available data are that risk factors such as blood urea nitrogen are not necessarily pathophysiologically linked to the development of the organ failure and, therefore, elevation of these markers simply reflects ongoing organ failure [9]. Theoretically, pathophysiologically relevant biomarkers may perform better in this clinical setting. In this regard, serum albumin may provide important prognostic information and help risk stratification. The advantage of using of serum albumin levels for predicting outcomes is that it is an objective variable which is easily performed, being inexpensive and available in most hospital laboratories [12].

The strengths of our study include the following: (i) this is the first study to investigate albumin not only as a risk factor but also as a predictor of persistent organ failure and mortality of acute pancreatitis by multivariable logistic regression analysis and subgroup analysis; (ii) exhaustive efforts were made to collect all clinical and laboratory data (including serum albumin and its related confounding factors) in the early stage of acute pancreatitis and a large sample with adequate power enabled us to evaluate the relationship between serum albumin and organ failure of acute pancreatitis; (iii) we have taken serum albumin levels within 24 hours of admission and excluded patients with albumin infusion before data collection so that artifactual influence on serum albumin levels due to intravenous fluid treatment would be minimized [55]. In addition, we also excluded patients with hypoalbuminemia due to malnutrition, chronic renal disease, albuminuria, bleeding/coagulation disorders, chronic alcoholism, hepatitis, and liver cirrhosis. Limitations of our study are as follows. (i) This is a retrospective study, which may have selection bias. Because of the retrospective design, we did not investigate other risk factors of severe acute pancreatitis such as lipase [56], prealbumin/fibrinogen [57], and red cell distribution width [58]. (ii) Data were collected in a single tertiary care hospital, which may not be generalized to a local clinic or a community hospital. Therefore, a prospective external validation of our results is mandatory before its application in the future. (iii) Serum albumin level may have been affected by fluid administration since it was measured within 24 hours of admission [12]. Therefore, another important limitation of current study is that we had no data on the volume of resuscitation or IV fluid therapy due to the retrospective study design. As a remediation, we have evaluated hematocrit and blood urea nitrogen, which often were used as the markers of hemoconcentration [59], as confounding factors of hypoalbuminemia in our multivariate logistic regression analysis.

In summary, as a risk factor, hypoalbuminemia within $24 \mathrm{hrs}$ of hospital admission is independently associated with increased risk of development of persistent organ failure and death in acute pancreatitis. An increase in serum albumin level was associated with a statistically significant reduction in the odds of persistent organ failure and mortality of acute pancreatitis after adjusting for potential confounders. The association between the serum albumin level and mortality was stronger in subgroups of patients with one day from pain onset to admission, nonbiliary etiology, and chronic concomitant diseases than in those subgroups with two or three days from pain onset to admission and biliary etiology and without chronic concomitant diseases. As a predictor, low albumin had a high negative predictive value and allowed identification of patients not at risk of developing persistent organ failure, thereby eliminating the need for intensive care.

\section{Ethical Approval}

This study protocol was approved by the Ethic Committee of the First Affiliated Hospital of Wenzhou Medical University. This study was performed according to the principles expressed in the Declaration of Helsinki.

\section{Consent}

Informed consent was obtained from the subjects.

\section{Conflicts of Interest}

The authors declare that they have no potential conflicts of interest or financial ties to declare.

\section{Authors' Contributions}

Wandong Hong joined in the design of the study and carried out the studies; Wandong Hong, Suhan Lin, Wujun Geng, and Jingye Pan participated in data collection. Wandong Hong conducted data analysis and drafted the manuscript. Maddalena Zippi, Simon Stock, Zarrin Basharat, Bicheng Cheng, and Mengtao Zhou helped to finalize the manuscript. All of the authors read and approved the manuscript. Wandong Hong, Wujun Geng, Bicheng Cheng, and Jingye Pan contributed equally to this work.

\section{References}

[1] P. G. Lankisch, M. Apte, and P. A. Banks, "Acute pancreatitis," The Lancet, vol. 386, no. 9988, pp. 85-96, 2015.

[2] R. Mounzer, C. J. Langmead, B. U. Wu et al., "Comparison of existing clinical scoring systems to predict persistent organ failure in patients with acute pancreatitis," Gastroenterology, vol. 142, no. 7, pp. 1476-1482, 2012.

[3] P. A. Banks, T. L. Bollen, C. Dervenis et al., "Classification of acute pancreatitis-2012: revision of the Atlanta classification and definitions by international consensus," Gut, vol. 62, no. 1, pp. 102-111, 2013.

[4] W. Hong, L. Dong, Q. Huang, W. Wu, J. Wu, and Y. Wang, "Prediction of severe acute pancreatitis using classification and regression tree analysis," Digestive Diseases and Sciences, vol. 56, no. 12, pp. 3664-3671, 2011.

[5] B. U. Wu, R. S. Johannes, X. Sun, Y. Tabak, D. L. Conwell, and P. A. Banks, "The early prediction of mortality in acute pancreatitis: a large population-based study," Gut, vol. 57, no. 12, pp. 1698-1703, 2008.

[6] P. G. Lankisch, B. Weber-Dany, K. Hebel, P. Maisonneuve, and A. B. Lowenfels, "The Harmless Acute Pancreatitis Score: A Clinical Algorithm for Rapid Initial Stratification of Nonsevere Disease," Clinical Gastroenterology and Hepatology, vol. 7, no. 6, pp. 702-705, 2009.

[7] E. Koutroumpakis, B. U. Wu, O. J. Bakker et al., "Admission hematocrit and rise in blood urea nitrogen at $24 \mathrm{~h}$ outperform 
other laboratory markers in predicting persistent organ failure and pancreatic necrosis in acute pancreatitis: a post hoc analysis of three large prospective databases," American Journal of Gastroenterology, vol. 110, no. 12, pp. 1707-1716, 2015.

[8] H. Nawaz, E. Koutroumpakis, J. Easler et al., "Elevated serum triglycerides are independently associated with persistent organ failure in acute pancreatitis," American Journal of Gastroenterology, vol. 110, no. 10, pp. 1497-1503, 2015.

[9] Y.-S. Peng, Y.-C. Chen, Y.-C. Tian et al., "Serum levels of apolipoprotein A-I and high-density lipoprotein can predict organ failure in acute pancreatitis," Critical Care, vol. 19, p. 88, 2015.

[10] M. A. Marinella and R. J. Markert, "Admission serum albumin level and length of hospitalization in elderly patients," Southern Medical Journal, vol. 91, no. 9, pp. 851-854, 1998.

[11] A. Gatta, A. Verardo, and M. Bolognesi, "Hypoalbuminemia," Internal and Emergency Medicine, vol. 7, no. 3, pp. 193-199, 2012.

[12] D. Viasus, C. Garcia-Vidal, A. Simonetti et al., "Prognostic value of serum albumin levels in hospitalized adults with communityacquired pneumonia," Journal of Infection, vol. 66, no. 5, pp. 415423, 2013.

[13] P. Goldwasser and J. Feldman, "Association of serum albumin and mortality risk," Journal of Clinical Epidemiology, vol. 50, no. 6, pp. 693-703, 1997.

[14] A. L. Holder, N. Gupta, E. Lulaj et al., "Predictors of early progression to severe sepsis or shock among emergency department patients with nonsevere sepsis," International Journal of Emergency Medicine, vol. 9, no. 1, article no. 10, pp. 1-11, 2016.

[15] M. E. Jellinge, D. P. Henriksen, P. Hallas, and M. Brabrand, "Hypoalbuminemia is a strong predictor of 30-day all-cause mortality in acutely admitted medical patients: a prospective, observational, cohort study," PLoS ONE, vol. 9, no. 8, Article ID e105983, 2014.

[16] O. Lyons, B. Whelan, K. Bennett, D. O'Riordan, and B. Silke, "Serum albumin as an outcome predictor in hospital emergency medical admissions," European Journal of Internal Medicine, vol. 21, no. 1, pp. 17-20, 2010.

[17] M. P. Holliday, D. Shaw, W. M. Thomas, and T. Leese, “Threshold for albumin as a prognostic marker in acute pancreatitis," British Journal of Surgery, vol. 76, no. 5, pp. 472-473, 1989.

[18] P. Xue, Z. W. Huang, Y. H. Li et al., "Clinical study on severe acute pancreatitis associated with hypoalbuminemia in early stage," Zhong Xi Yi Jie He Xue Bao, vol. 3, no. 6, pp. 443-445, 2005.

[19] A. Gonzalvez-Gasch, G. García de Casasola, R. Barba Martín, B. Herreros, and C. Guijarro, "A simple prognostic score for risk assessment in patients with acute pancreatitis," European Journal of Internal Medicine, vol. 20, no. 3, pp. e43-e48, 2009.

[20] S. Li, Y. Zhang, M. Li, C. Xie, and H. Wu, "Serum albumin, a good indicator of persistent organ failure in acute pancreatitis," BMC Gastroenterology, vol. 17, no. 1, 2017.

[21] W.-D. Hong, X.-R. Chen, S.-Q. Jin, Q.-K. Huang, Q.-H. Zhu, and J.-Y. Pan, "Use of an artificial neural network to predict persistent organ failure in patients with acute pancreatitis," Clinics, vol. 68, no. 1, pp. 27-31, 2013.

[22] A. Michaud, G. B. Marchand, M. Nadeau et al., "Biliopancreatic Diversion with Duodenal Switch in the Elderly: Long-Term Results of a Matched-Control Study," Obesity Surgery, vol. 26, no. 2, pp. 350-360, 2016.

[23] P. C. Schroy, J. B. Wong, M. J. O’Brien, C. A. Chen, and J. L. Griffith, "A risk prediction index for advanced colorectal neoplasia at screening colonoscopy," American Journal of Gastroenterology, vol. 110, no. 7, pp. 1062-1071, 2015.

[24] J. Peat, B. Barton, and E. Elliott, Statistics Workbook for EvidenceBased Healthcare, Blackwell, Malden, MA, USA, 2008.

[25] P. Royston, "PTREND: stata module for trend analysis for proportions," 2014, https://ideas.repec.org/c/boc/bocode/s426101 .html.

[26] J. Cuzick, "A wilcoxon-type test for trend," Statistics in Medicine, vol. 4, no. 1, pp. 87-90, 1985.

[27] G. Grimm, H. Haslacher, T. Kampitsch et al., "Sex differences in the association between albumin and all-cause and vascular mortality," European Journal of Clinical Investigation, vol. 39, no. 10, pp. 860-865, 2009.

[28] E. De-Madaria, P. A. Banks, N. Moya-Hoyo et al., "Early Factors Associated With Fluid Sequestration and Outcomes of Patients With Acute Pancreatitis," Clinical Gastroenterology and Hepatology, vol. 12, no. 6, pp. 997-1002, 2014.

[29] P. Mentula, M.-L. Kylänpää, E. Kemppainen et al., "Early prediction of organ failure by combined markers in patients with acute pancreatitis," British Journal of Surgery, vol. 92, no. 1, pp. 68-75, 2005.

[30] R. L. Grant, "Converting an odds ratio to a range of plausible relative risks for better communication of research findings," British Medical Journal, vol. 348, no. jan24 1, pp. f7450-f7450, 2014.

[31] W.-D. Hong, Q.-H. Zhu, Z.-M. Huang et al., "Predictors of esophageal varices in patients with HBV-related cirrhosis: a retrospective study," BMC Gastroenterology, vol. 9, article 11, 2009.

[32] M. Maksimow, L. Kyhälä, A. Nieminen et al., "Early prediction of persistent organ failure by soluble CD73 in patients with acute pancreatitis*," Critical Care Medicine, vol. 42, no. 12, pp. 25562564, 2014.

[33] J.-L. Vincent, J. A. Russell, M. Jacob et al., "Albumin administration in the acutely ill: What is new and where next," Critical Care, vol. 18, no. 6, article no. 630, p. 231, 2014.

[34] Y.-J. Zheng, B. Zhou, G. Ding et al., "Effect of serum from patients with severe acute pancreatitis on vascular endothelial permeability," Pancreas, vol. 42, no. 4, pp. 633-639, 2013.

[35] C. Uhlig, P. L. Silva, S. Deckert, J. Schmitt, and M. G. de Abreu, "Albumin versus crystalloid solutions in patients with the acute respiratory distress syndrome: a systematic review and metaanalysis," Critical Care, vol. 18, no. 1, article R10, 2014.

[36] G. J. Quinlan, S. Mumby, G. S. Martin, G. R. Bernard, J. M. C. Gutteridge, and T. W. Evans, "Albumin influences total plasma antioxidant capacity favorably in patients with acute lung injury," Critical Care Medicine, vol. 32, no. 3, pp. 755-759, 2004.

[37] C.-W. Chen, Y.-Y. Chen, C.-L. Lu et al., "Severe hypoalbuminemia is a strong independent risk factor for acute respiratory failure in COPD: A nationwide cohort study," International Journal of COPD, vol. 10, pp. 1147-1154, 2015.

[38] T. B. Horwich, K. Kalantar-Zadeh, R. W. MacLellan, and G. C. Fonarow, "Albumin levels predict survival in patients with systolic heart failure," American Heart Journal, vol. 155, no. 5, pp. 883-889, 2008.

[39] C. J. Wiedermann, W. Wiedermann, and M. Joannidis, "Causal relationship between hypoalbuminemia and acute kidney injury," World Journal of Nephrology, vol. 6, no. 4, p. 176, 2017.

[40] M. A. Kaufmann, I. Castelli, H. Pargger, and L. J. Drop, "Nitric oxide dose-response study in the isolated perfused rat 
kidney after inhibition of endothelium-derived relaxing factor synthesis: the role of serum albumin," Journal of Pharmacology and Experimental Therapeutics, vol. 273, no. 2, pp. 855-862, 1995.

[41] E. H. Lee, S. H. Baek, and J. H. Chin, "Preoperative hypoalbuminemia is a major risk factor for acute kidney injury following off-pump coronary artery bypass surgery," Intensive Care Medicine, vol. 38, no. 9, pp. 1478-1486, 2012.

[42] R. Dixon and N. J. Brunskill, "Activation of mitogenic pathways by albumin in kidney proximal tubule epithelial cells: implications for the pathophysiology of proteinuric states," Journal of the American Society of Nephrology, vol. 10, no. 7, pp. 1487-1497, 1999.

[43] J. Iglesias, V. E. Abernethy, Z. Wang, W. Lieberthal, J. S. Koh, and J. S. Levine, "Albumin is a major serum survival factor for renal tubular cells and macrophages through scavenging of ROS," The American Journal of Physiology-Renal Physiology, vol. 277, no. 5, pp. F711-F722, 1999.

[44] P. Caironi, G. Tognoni, and L. Gattinoni, "Albumin replacement in severe sepsis or septic shock.", The New England journal of medicine, vol. 371, no. 1, p. 84, 2014.

[45] F. Salerno, R. J. Navickis, and M. M. Wilkes, "Albumin treatment regimen for type 1 hepatorenal syndrome: A dose-response meta-analysis," BMC Gastroenterology, vol. 15, no. 1, article no. 167, 2015.

[46] F. Salerno, R. J. Navickis, and M. M. Wilkes, "Albumin infusion improves outcomes of patients with spontaneous bacterial peritonitis: a meta-analysis of randomized trials," Clinical Gastroenterology and Hepatology, vol. 11, no. 2, pp. 123-130, 2013.

[47] P. G. Wilson, M. Manji, and J. P. Neoptolemos, "Acute pancreatitis as a model of sepsis," Journal of Antimicrobial Chemotherapy, vol. 41, pp. 51-63, 1998.

[48] C. Xu, Z. Qiao, Y. Lu et al., "Influence of fatty liver on the severity and clinical outcome in acute pancreatitis," PLOS ONE, vol. 10, no. 11, Article ID e0142278, 2015.

[49] I. Mikolasevic, L. Orlic, G. Poropat et al., "Nonalcoholic fatty liver and the severity of acute pancreatitis," European Journal of Internal Medicine, vol. 38, pp. 73-78, 2017.

[50] J. H. Cho, T. N. Kim, and S. B. Kim, "Comparison of clinical course and outcome of acute pancreatitis according to the two main etiologies: alcohol and gallstone," BMC Gastroenterology, vol. 15, no. 1, article no. 87, 2015.

[51] B. U. Wu, O. J. Bakker, G. I. Papachristou et al., "Blood urea nitrogen in the early assessment of acute pancreatitis: an international validation study," Archives of Internal Medicine, vol. 171, no. 7, pp. 669-676, 2011.

[52] W. Hong, S. Lin, M. Zippi et al., "High-density lipoprotein cholesterol, blood urea nitrogen, and serum creatinine can predict severe acute pancreatitis," BioMed Research International, vol. 2017, pp. 1-7, 2017.

[53] S. Lin, W. Hong, Z. Basharat, Q. Wang, J. Pan, and M. Zhou, "Blood urea nitrogen as a predictor of severe acute pancreatitis based on the revised atlanta criteria: timing of measurement and cutoff points," Canadian Journal of Gastroenterology and Hepatology, vol. 2017, Article ID 9592831, 7 pages, 2017.

[54] A. Kumaravel, T. Stevens, G. I. Papachristou et al., "A Model to Predict the Severity of Acute Pancreatitis Based on Serum Level of Amylase and Body Mass Index," Clinical Gastroenterology and Hepatology, vol. 13, no. 8, pp. 1496-1501, 2015.

[55] D. B. Gundpatil, B. L. Somani, T. K. Saha, and M. Banerjee, "Serum Urea: Albumin ratio as a prognostic marker in critical patients with non-chronic kidney disease," Indian Journal of Clinical Biochemistry, vol. 29, no. 1, pp. 97-100, 2014.

[56] V. V. Gumaste, P. B. Dave, D. Weissman, and J. Messer, "Lipase/amylase ratio. A new index that distinguishes acute episodes of alcoholic from nonalcoholic acute pancreatitis," Gastroenterology, vol. 101, no. 5, pp. 1361-1366, 1991.

[57] W. Yue, Y. Liu, W. Ding et al., "The predictive value of the prealbumin-to-fibrinogen ratio in patients with acute pancreatitis," International Journal of Clinical Practice, vol. 69, no. 10, pp. 1121-1128, 2015.

[58] D. Wang, J. Yang, J. Zhang et al., "Red cell distribution width predicts deaths in patients with acute pancreatitis," Journal of Research in Medical Sciences, vol. 20, no. 5, pp. 424-428, 2015.

[59] G. A. Cote, S. V. Sagi, S. E. Schmidt et al., "Early measures of hemoconcentration and inflammation are predictive of prolonged hospitalization from post - Endoscopic retrograde cholangiopancreatography pancreatitis," Pancreas, vol. 42, no. 5, pp. 850-854, 2013. 


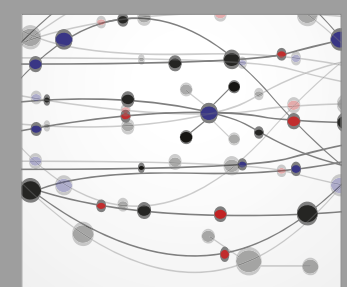

The Scientific World Journal
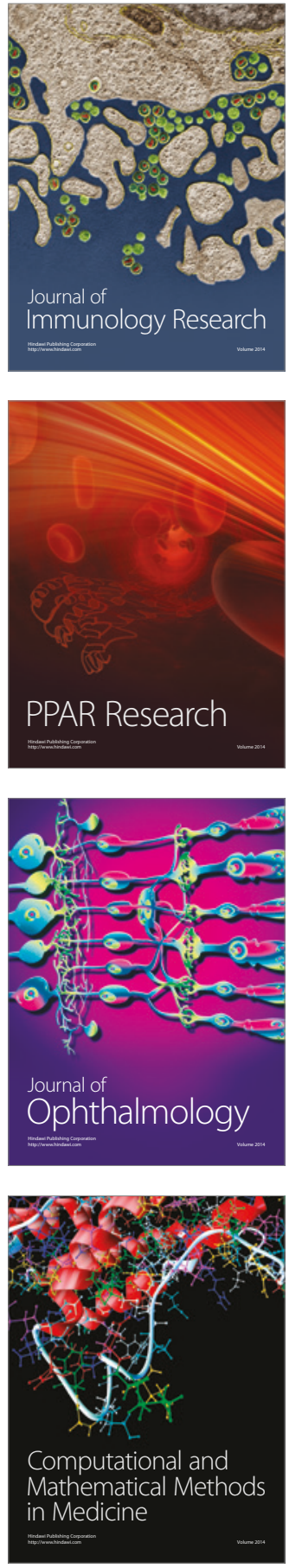

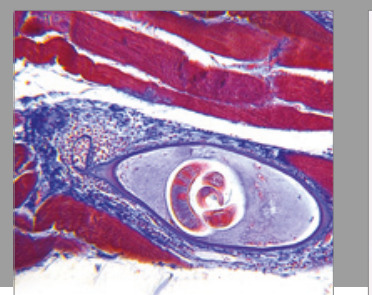

Gastroenterology Research and Practice
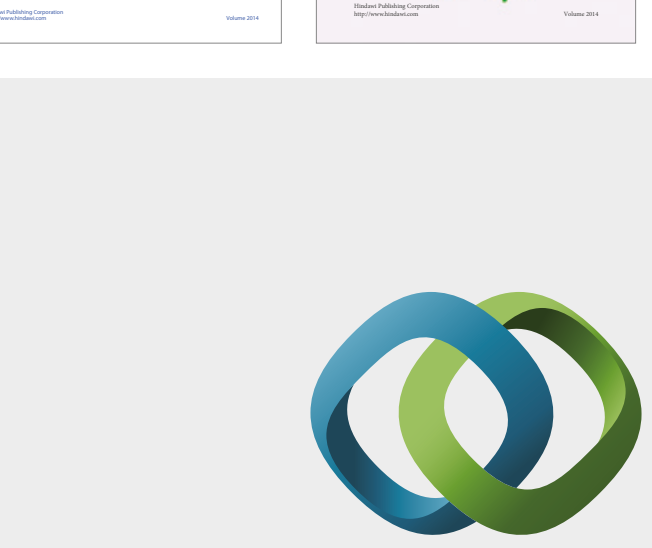

\section{Hindawi}

Submit your manuscripts at

https://www.hindawi.com
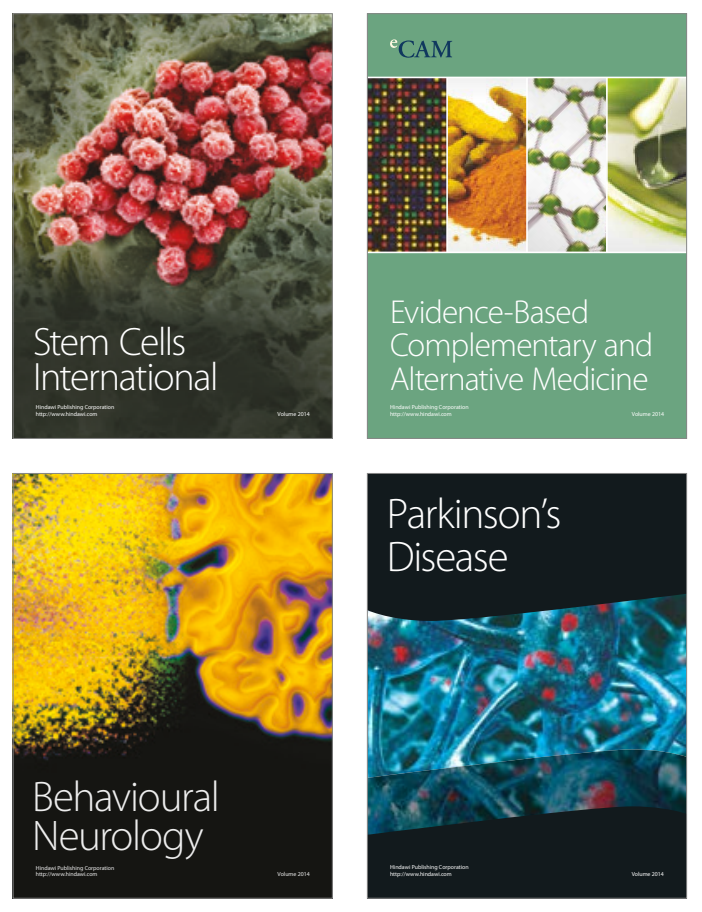
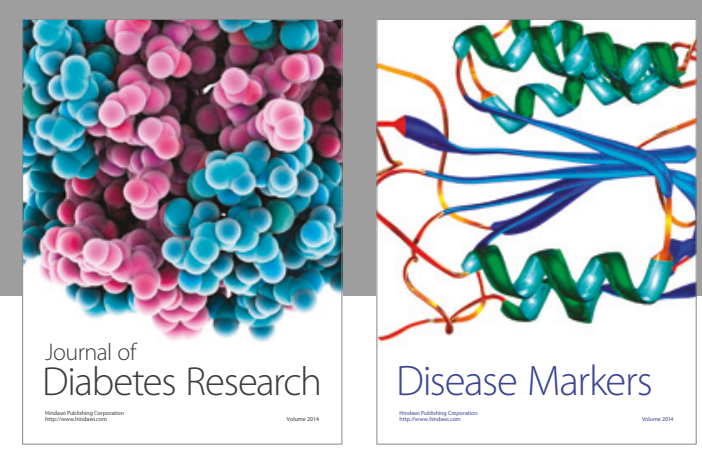

Disease Markers
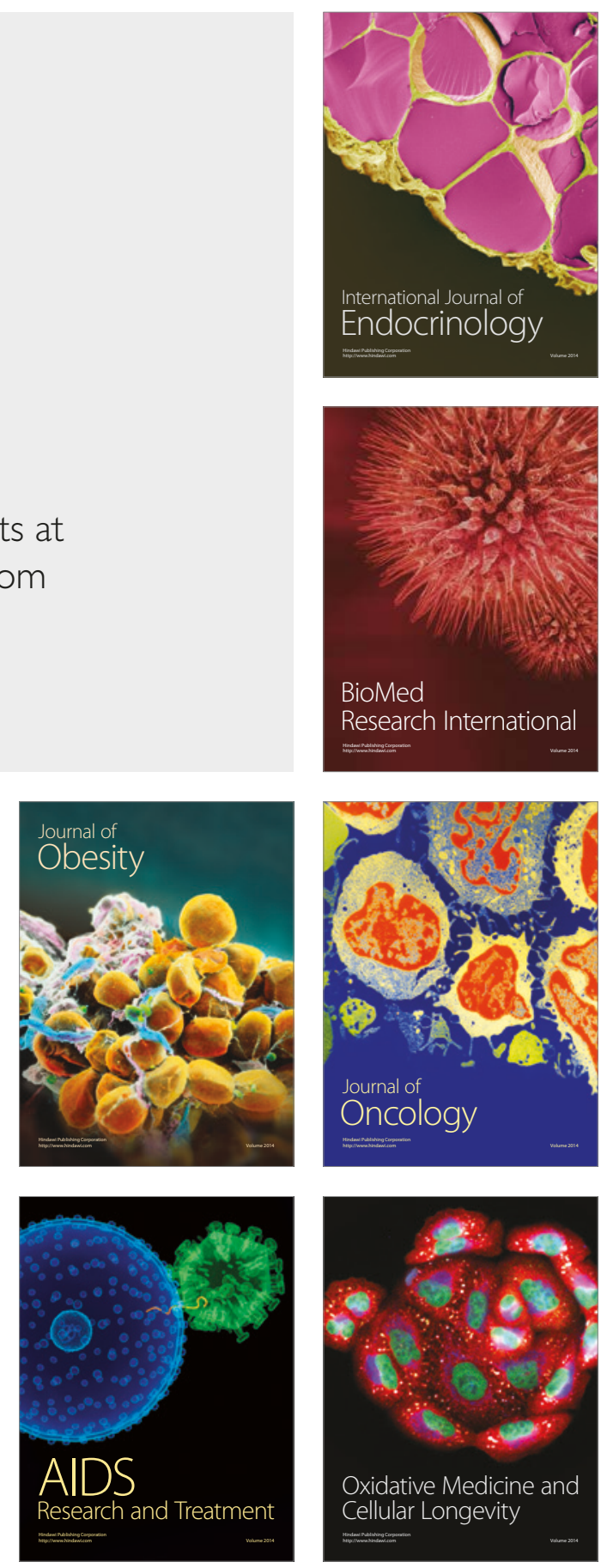